\title{
Mejora del impacto mediante difusión de la investigación en redes sociales: \#PublicaSalutlB
}

\author{
Elena Pastor-Ramon* \\ Virgili Páez*
}

Artículo recibido:

5 de octubre de 2020

Artículo aceptado:

26 de febrero de 2021

Artículo de investigación

\section{Resumen}

Objetivo. El principal objetivo de la biblioteca es facilitar el acceso a la información a sus usuarios. Las bibliotecas científicas deben también centrarse en la contribución científica y en los resultados de la investigación de sus instituciones.

Metodología. En el Sistema Sanitario Público de las Illes Balears hay 16.300 profesionales de diferentes categorías, que trabajan en siete hospitales y 56 centros de atención primaria. La Biblioteca Virtual de Ciencias de la Salud de las Illes Balears (Bibliosalut), creada en 2003 (Páez et al., 2013; Páez Cervi, 2005), empezó a tener presencia en redes sociales en 2012 (Pastor-Ramon, Páez y Sastre-Suárez, 2014). Desde

* Biblioteca Virtual de Ciencias de la Salud de las Islas Baleares (Bibliosalut). Consejería de Salud y Consumo, España_ epastor.bibliosalut@gmail.com vpaez@bibliosalut.com

INVESTIGACIÓN BIBLIOTECOLÓGICA, vol. 35, núm. 88, julio/septiembre, 2021, México, ISSN: 2448-8321 pp. 29-44 
un principio se quisieron difundir los resultados de la investigación de los profesionales del Servicio Sanitario Público de las Illes Balears, utilizando para ello el hashtag \#PublicaSalutIB. Con esta etiqueta se quería dar la opción de que cualquier investigador de las Illes Balears pudiera utilizarla, independientemente del centro en el que trabajara.

Conclusiones. Este servicio mejora la relación usuario-biblioteca, ya que los autores conocen su utilidad y la visibilidad que da a sus investigaciones. En más de ocho años se ha dado difusión, a través de las redes sociales, a más de 2.900 documentos y, desde 2015, se añadió la publicación de una noticia en el portal www. infosalut.com, un producto de Bibliosalut.

Al brindar una información de la referencia bibliográfica y su difusión en redes sociales conseguimos, además, que se genere un impacto inmediato, que puede ser medido gracias a métricas complementarias como Altmetric y PlumX, lo que también puede influir en la valoración que recibe cada trabajo.

Palabras clave: SEOA; SEO Académico; Redes Sociales; Bibliotecas Virtuales; Hashtags; Investigación Sanitaria; Herramientas de Gestión de Redes Sociales

Improving the impact by disseminating research on social networks: \#PublicaSalutIB

Elena Pastor-Ramon and Virgili Páez

\section{Abstract}

Objectives. The main objective of the library is to facilitate access to information for its users. However scientific libraries should also focus on the scientific contribution and research results of their institutions. Methodology. There are 16,300 professionals from different categories in the Balearic Islands Public Health System, working in 7 hospitals and 56 primary care centres. The Balearic Islands Virtual Health Sciences Library (Bibliosalut), created in 2003 (Páez et al., 2013; Páez Cervi, 2005), began to have presence on social networks in 2012 (Pastor-Ramon, Páez, and Sastre-Suárez, 2014). From the outset the aim was to disseminate the results of research carried out by professionals from 
the Balearic Islands Public Health Service, using the hashtag \#PublicaSalutIB. Thus the aim of this hashtag was to give any researcher on the Balearic Islands the option of using it, regardless of the centre where they worked in.

Conclusions. This service improves the user-library relationship, since authors know how useful it is and how much visibility it gives to their research. In a little more than 8 years, more than 2,900 documents have been disseminated through social networks and, since 2015, a news item has been added to the Bibliosalut portal for each one of the new documents: www.infosalut.com. By providing information about the bibliographical reference and disseminating it on social networks, we also achieve an immediate impact, which can be measured thanks to complementary metrics such as Altmetric and PlumX, which can also influence the evaluation that each work receives.

Keywords: ASEO; Academic Scientific Engine Optimization; Social Networks; Virtual Libraries; Hashtags; Health Research; Social Networks Managing Tools

\section{INTRODUCCIÓN}

— n los últimos 18 años la evolución de la biblioteca de ciencias de la salud de las Illes Balears (España) ha sido notable, en muchas ocasiones de la mano de su adaptación al entorno digital, motivo por el cual presentar algunos de los motores de este cambio puede ser de utilidad tanto para profesionales de otras bibliotecas como para investigadores (Costa Marín et al., 2011; Páez, 2019).

Podemos situar el cambio producido en Baleares en 2003, cuando se empezaron a centralizar las suscripciones, aunque la centralización formal de los servicios se realizó en 2008 (Páez, 2019; Páez et al., 2013). Durante esos años, además, las redes sociales fueron evolucionando y tomando cada vez más protagonismo en la sociedad, en prácticamente todas sus facetas. Facebook se crea en 2004 (Facebook, 2020), Twitter en 2006, y en 2008 llegó el impacto en la publicación de investigaciones españolas sobre su aplicación en bibliotecas (Margaix-Arnal, 2008; Peset, Ferrer-Sapena y Baiget, 2008).

Es en 2012 cuando se decide utilizar las redes sociales como vía de comunicación entre la Biblioteca Virtual de Ciències de la Salut de les Illes Balears (Bibliosalut) y los usuarios potenciales del Sistema Sanitario Público de las 
Illes Balears (SSPIB) (Pastor-Ramon, Páez y Sastre-Suárez, 2014). Ese año se decide que la biblioteca tendría un perfil propio en Twitter, Facebook, SlideShare, Flickr y el emergente Google+ (ahora desactivado). Para dar este paso, se tuvieron en cuenta diversos principios:

- Constancia en la publicación. No se podía empezar a publicar y dar actividad a las cuentas, y después ir alargando los días de publicación hasta dejar los perfiles incativos, como sucede en algunas bibliotecas.

- Diversidad. Se debía dar visibilidad a los diferentes servicios de Bibliosalut.

- Utilidad. Se debían proponer recursos e informaciones interesantes para los usuarios potenciales de la biblioteca, ya fuera respecto a nuevas guías de práctica clínica, sugerencias de artículos o compartir información que otras bibliotecas estaban difundiendo y también podrían ser interesantes para los usuarios de Bibliosalut.

- Apoyo en las redes. La idea era utilizar las plataformas digitales, en especial redes sociales, para dar difusión a la producción científica de los investigadores de la comunidad de las Illes Balears.

Es en este último punto en el que vamos a centrar este trabajo. En concreto, presentaremos los resultados del uso de Twitter y Facebook para la difusión de la producción científica, utilizando hashtags. En el caso que vamos a presentar, utilizamos concretamente la etiqueta \#PublicaSalutIB.

Un hashtag o etiqueta es, según Fundéu, "una palabra, frase o grupo de caracteres alfanuméricos que se emplea en las redes sociales para agrupar varios mensajes sobre un mismo tema; se identifica fácilmente, ya que está compuesto por el símbolo \# (hash) y un nombre o etiqueta (tag)" (Fundéu BBVA, 2017).

Con el uso de esta etiqueta se quería unificar la información relacionada con los artículos, cartas, tesis doctorales, comunicaciones en congresos y, en general, la producción científica de los profesionales del SSPIB, que hasta ese momento no era difundida a través de redes sociales y, por lo tanto, su impacto dependía únicamente del azar a la hora de hacer una búsqueda bibliográfica.

El objetivo era que cualquier profesional -investigador de la Universidad de las Illes Balears, de un centro privado u otra organización- que hubiese publicado una investigación relacionada con salud en las Illes Balears, independientemente de que fuese profesional del SSPIB, pudiera hacer uso de esta etiqueta para difundir su trabajo. 


\section{Metodología}

En 2012 se inició el proyecto \#PublicaSalutIB, realizando unas búsquedas de las que se guardaron las RSS (Really Simple Syndication), configuradas en diferentes bases de datos, con la filiación de cada uno de los centros de las Illes Balears, así como las diferentes firmas posibles. Esta parte fue la más complicada porque muchos de los centros no tenían unificados sus nombres y podían quedar algunos artículos fuera de esta búsqueda.

Además, los centros que pertenecen al SSPIB podían publicar artículos en coautoría con otros centros, como la Universidad de las Illes Balears, por lo que este centro no se podía eliminar automáticamente. Así que, después de configurar todas estas RSS en un gestor como Feedly, era necesario ir comprobando las firmas (Hospital Universitario Son Espases, Hospital Universitario Son Llàtzer, Hospital Can Misses, Hospital Mateu Orfila, Atención Primaria de Mallorca, Hospital Comarcal de Inca, Hospital de Manacor, Hospital de Formentera, diferentes centros de salud de Baleares, Instituto de Investigación Sanitaria Illes Balears, Consejería de Salud, IB-Salut, Área de Salud de Menorca o el Área de Salud de Ibiza y Formentera) para eliminar aquellas referencias que no fuesen de un centro del SSPIB, cuya dirección hiciese relación a alguna de las poblaciones de las Illes Balears (calle Islas Baleares, Ibiza o Mallorca, por ejemplo) y aquellos centros que fuesen de gestión privada o únicamente de la Universidad de las Illes Balears.

Después de realizar la selección y tener claras cuáles eran las referencias pertenecientes a investigadores de Balears, éstas se guardaban en un gestor bibliográfico para ir haciendo las noticias; una vez hecha la noticia se guarda en una carpeta de publicados. Al principio se iba guardando por centros, pero hace unos años se unieron todas las referencias en la misma carpeta pues no se vio necesaria esta separación. Primero se gestionaban con Refworks y en estos momentos se utiliza Mendeley.

Los tres primeros años sólo se hacía un tuit o un post de Facebook y Google + con la información del servicio que había escrito el documento, se etiquetaba a la institución si ésta tenía presencia en las redes sociales y después se ponía la URL configurada para el resolvedor de enlaces de Bibliosalut. De esta forma quien entrase podría ver si tenía acceso al texto completo o solicitarlo directamente al Servicio de Obtención de Documentos de Bibliosalut.

El 24 de enero de 2012 se publicó el primer tuit y post de Facebook (Figuras 1 y 2), utilizando el hashtag \#PublicaSalutIB. 
Bibliosalut @bibliosalut·24 gen. 2012

\#PublicaSalutlB Effect of Co-Inheritance of Beta-Thalassemia and Hemochromatosis Mutations on Iron Overload 1.usa.gov/x2xoXo

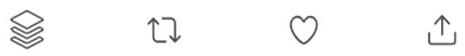

Figura 1. Primer tuit con el hashtag \#PublicaSalutlB

\section{Bibliosalut}

Página A 514 personas les gusta esto Sitio web de salud y bienestar

24 ene. 2012 - 3. \#PublicaSalutIB Effect of Co-Inheritance of BetaThalassemia and Hemochromatosis Mutations on Iron Overload http://1.usa.gov/x2xoXo

Figura 2. Primer posten Facebook con el hashtag \#PublicaSalutlB

Unos meses después de empezar a utilizar este hashtag, se detectó que diferentes bibliotecas virtuales españolas empezaban a utilizar también un hashtag para etiquetar la producción científica de sus investigadores. Así se vio que@BibliotecaSalut, de Catalunya, utilizaba \#publicaDSalut (10/08/2012); Bibliosaúde, de Galicia, \#PublicaSergas (06/08/2014); BVMurcia, de Murcia, \#PubSaludMurcia (16/08/2017), y Bibliosalud, de Aragón, \#InvestigaSaludAragon (26/10/2016).

Se sabe que otras bibliotecas de centros sanitarios también han creado su hashtag, pero al no tratarse de bibliotecas virtuales de salud autonómicas no las hemos tenido en cuenta en este trabajo.

Es en abril de 2015 cuando se crea el portal de noticias infosalut.com (Páez et al., 2016), un servicio de Bibliosalut, y entonces se decide que, además de hacer difusión de un documento, se haría también una noticia con información sobre los autores pertenecientes al SSPIB, la institución a la que pertenecen, otros centros no SSPIB, información de la revista y una traducción del resumen del inglés al catalán y castellano, si éste está disponible en la base de datos. También se incluyeron los datos de la referencia bibliográfi$\mathrm{ca}$, enlaces de Bibliosalut para poder consultar el texto completo si la revista pertenece a la colección de la biblioteca, o solicitarlo al Servicio de Obtención de Documentos (Figura 3). En este momento, se decidió dejar de utilizar Google+, debido a su escaso uso por los usuarios de redes sociales. 


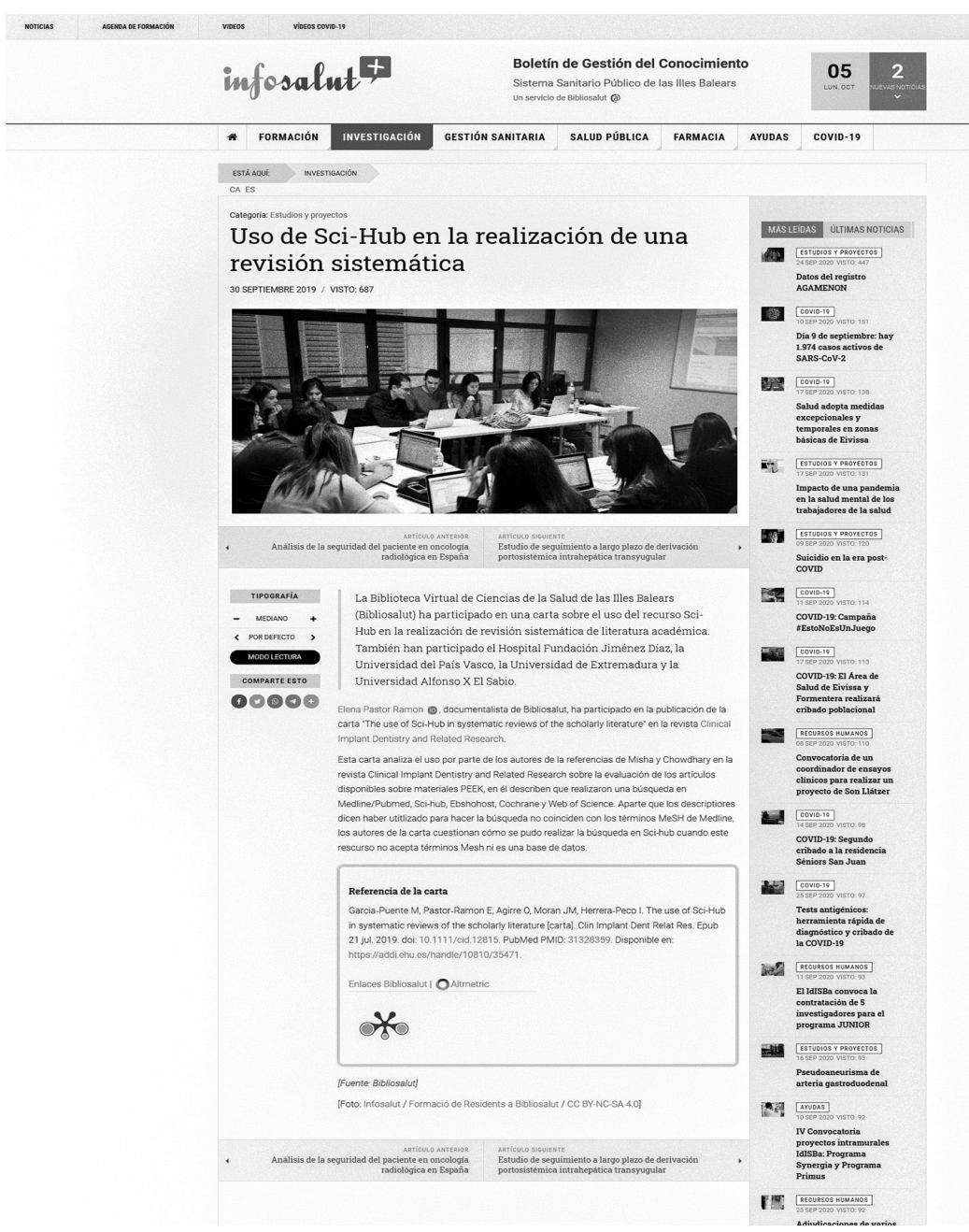

Figura 3. Noticia en Infosalut de un artículo publicado por una investigadora del SSPIB

Desde 2015 se comparte la noticia a través de Twitter y Facebook en las cuentas de Infosalut, y una semana más tarde se comparte en las cuentas de Bibliosalut. De esta forma podemos alargar la promoción de la publicación. El formato del tuit es el siguiente (Figura 4):

\#PublicaSalutIB Título de la noticia en Infosalut.com, URL de la noticia, usuario de Twitter de autor/es, institución, URL DOI o PubMed o texto completo, si éste está disponible en open access, instituciones o sociedades profesionales de las Illes Balears que podrían estar interesados en este artículo. 
@infosalut
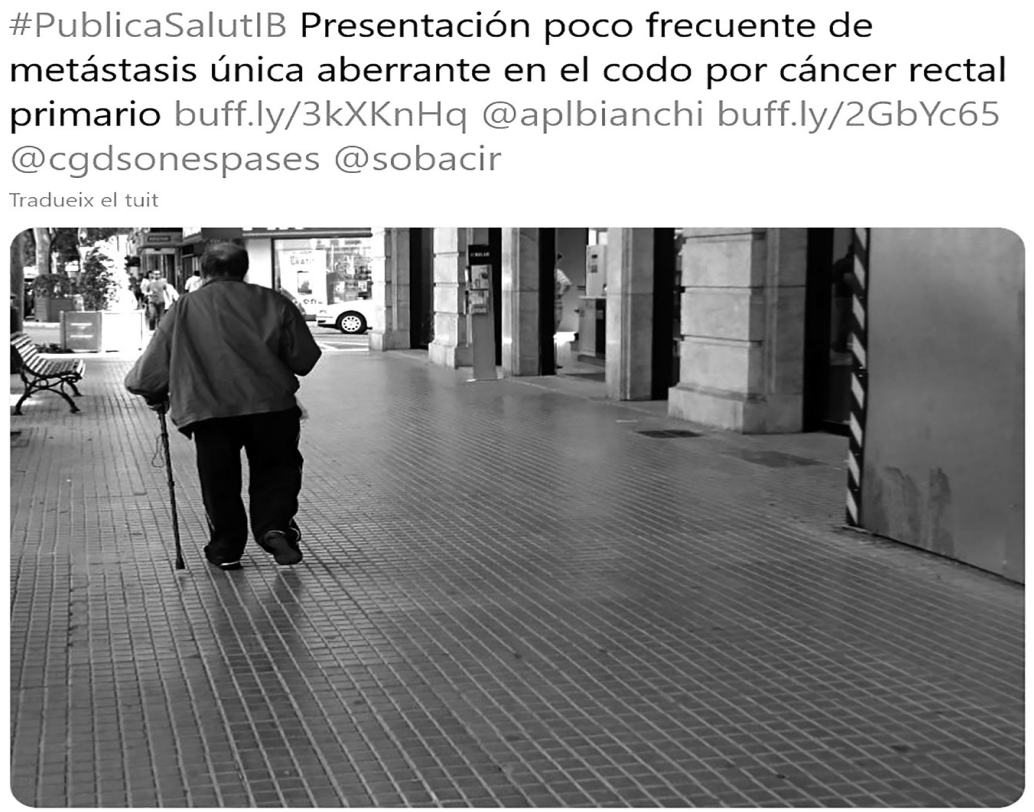

6:05 p. m. 29 de set. de $2020 \cdot$ Buffer

Figura 4. Ejemplo de tuit en la actualidad con el hashtag\#PublicasalutIB

Desde 2012 se han compartido en Bibliosalut 2.900 referencias a través de Twitter y en Infosalut, y desde abril de 2015 se han redactado más de 1.900 noticias relacionadas con artículos, comunicaciones en congresos o tesis doctorales.

\section{ANÁLISIS DE DATOS}

Durante estos años se ha hecho difusión de una gran cantidad de documentos, como las comunicaciones de congresos o tesis doctorales, que en condiciones normales o no hubiesen sido difundidos, o bien sin una aportación sobre las comunicaciones y pósteres presentados.

Muchos investigadores pertenecientes al SSPIB se han dado cuenta de la visibilidad que les da la publicación de la noticia en infosalut.com y comunican la publicación de los artículos o la asistencia a congresos, ya sea por correo electrónico, etiquetando a Bibliosalut o Infosalut en un tuit, por mensaje directo a través de Twitter o por un formulario dentro de la web de Bibliosalut, a través del Servicio de Atención al Usuario "Pregunta a Bibliosalut". 
Además, se puede comprobar en el vínculo que se añade a cada artículo del que se hace la noticia que aparecen tanto Bibliosalut como Infosalut como emisores del tuit, y eso da una puntuación a la publicación. El problema viene cuando el artículo no tiene DOI y no está presente en MEDLINE, porque la revista no lo tiene, y no genera ningún impacto en redes sociales, a pesar de que se haga esta difusión. Este es un tema a ser analizado aparte: la importancia de disponer de datos como el DOI para que un artículo pueda ser rastreado.

Para poder demostrar la importancia de este servicio en el impacto de los artículos, en este caso en el número de referencias con citas, se realizó un análisis de los artículos publicados por investigadores del Sistema Sanitario de las Illes Balears en revistas indexadas en la Web of Science. Lo que se quería analizar era que en el momento en el que un artículo tuviese como mínimo un 1 de puntuación Altmetric, quería decir que se había hecho difusión a través de redes sociales, independientemente de que lo hubiese hecho Bibliosalut, alguno de los autores o la institución.

En enero de 2020 se realizó una búsqueda en la que se intentó obtener el máximo número de referencias de los artículos publicados entre 2012 y 2019 (se incluyeron todos los topónimos posibles de las Illes Balears), y una vez aplicado este límite se procedió a limitar por materia. Al principio se miró por centro, pero se tenía la sensación de que faltaba información. Una vez aplicados estos filtros se fueron guardando los archivos por años tanto en un formato para poder ser subido a un gestor bibliográfico (en formato ris) como en Excel, para poder analizar los datos.

Los archivos incluyen información de las citas: autores, título, revista, año de publicación, volumen, número, DOI, número de citas. La información que interesaba analizar era el número de citas y cruzarla con el número de Altmetric y citas que nos daba Dimensions, el resto no era de utilidad para este análisis.

Cuando se descargó la información de las referencias, se eliminaron los documentos que no tenían campo DOI o PMID (PubMed Identifier), ya que no podía ser detectada su difusión.

Las referencias finales obtenidas para ser analizadas sumaron 2.693, entonces se procedió a realizar la búsqueda de esos DOI o PMID (ya que todos tenían uno u otro dato) en la base de datos Explorer de Altmetric; esta base de datos da información completa sobre la difusión de un artículo a través de redes sociales.

En esta base de datos podemos realizar una búsqueda utilizando una estrategia de PubMed. De esta forma fue más sencillo ya que sólo se tenía que añadir la etiqueta [DOI] o [PMID] después de cada identificador: 
De cada una de las búsquedas se descargaron los archivos en formato cvs. Tanto los datos de la Web of Science como los de Explorer de Altmetric se unificaron en un archivo de Excel, separando los datos en diferentes hojas para poder ser analizados.

Para poder combinar los datos de las diferentes hojas dentro del mismo archivo de Excel se utilizó la fórmula siguiente:

$=\operatorname{SIND}(\operatorname{SIERROR}(\mathrm{CONSULV}(M 2 ; d o i ! \$ A \$ 1: \$ A K \$ 3553 ; 2 ; \mathrm{FALS}) ; \operatorname{CONSULV}(\mathrm{N} 2 ;$ pmid!\$A $\$ 1: \$-$

T\$3553;2;FALS));0)

De esta forma, lo que se conseguía era recuperar los artículos que coincidiesen en los datos de PMID o DOI, tanto en la hoja Altmetric como en la de los datos de la Web of Science, y se añadiría la puntuación de Altmetric en la columna correspondiente. Los artículos que no tuviesen ese dato (aparece con el dato N/D) tendrían un 0 , porque significaría que no se ha hecho difusión en redes sociales. Una vez recuperados todos estos datos se realizó una prueba $\mathrm{T}$, se comparó la variable de número de citas recibidas en la Web of Science con la difusión realizada en redes sociales, en la que se vio que los artículos de los que la columna Altmetric estaba a 0, por lo que se puede pensar que no se había realizado difusión a través de redes sociales o con presencia en los recursos que analiza Altmetric, tenían una media de 1,62, mientras que los que tenían dato de Altmetric tenían una media de 3,827 ( Figura 5). Creemos que los datos nos muestran que se puede asociar un aumento de citas en aquellos artículos en los que se ha realizado algún tipo de difusión. Aunque el archivo que nos ofrece el Explorer de Altmetric da más información de dónde se ha compartido una referencia, el análisis se limitó a la correlación de citas y puntuación de Altmetric, ya que se quería conocer la difusión en general, no en recursos en concreto.

\section{Discusión}

El uso de las redes sociales por parte de la institución para hacer difusión de la producción científica se puede considerar parte del denominado Academic Search Engine Optimization (ASEO), que no sólo asegura que los artículos se encuentren, rastreen e indicen, sino que tiene un gran efecto con la intención de que los artículos se muestren (Green, 2017). Dentro de este proceso también se incluye el uso de redes sociales, como último paso en la producción científica (Ramírez Vega, 2018). 


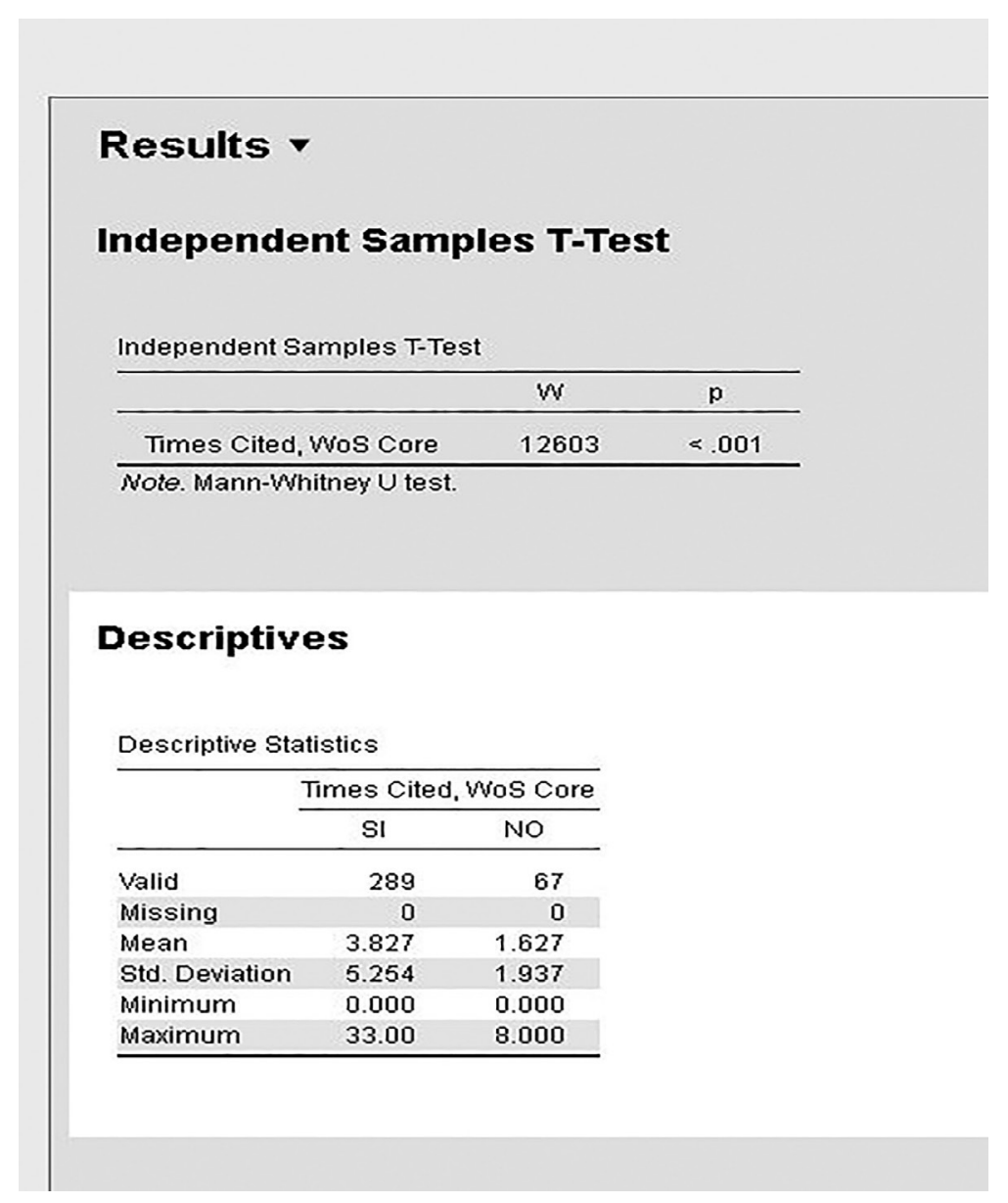

Figura 5. Prueba T con las citas de la wos y la puntuación de Almetric

Lo que se pretende con esta difusión, además de que se conozca la investigación, es que la institución gane en influencia o autoridad gracias al impacto de la producción científica hecha en su nombre. Diferentes estudios demuestran que una difusión de la investigación a través de un blog o participando en conversaciones de Twitter puede hacer que aumente el número de descargas y lecturas (Allen et al., 2013; Buckarma et al., 2017; Choo et al., 2015).

El impacto más inmediato se puede medir gracias a las llamadas métricas alternativas. En 2010 se empezó a hablar del concepto métricas alternativas, o Altmetrics. Uno de los primeros en acuñar el término fue Jason Priem, en un tuit lanzado el 29 de septiembre de 2010: "I like the term \#articlelevelmetrics, but it fails to imply *diversity* of measures. Lately, I'm liking \#altmetrics". 
Según la National Information Standards Organization (NISO) (Carpenter, Lagace, y Bahnmaier, 2016), se trata de un "término amplio que encapsula la colección de diferentes indicadores digitales relacionados con trabajos académicos. Estos indicadores se derivan de la actividad y compromiso entre diversas partes interesadas y resultados académicos en el ecosistema de la investigación, incluyendo la esfera pública".

Estas métricas nos ofrecen un panorama sobre la difusión que se está haciendo en redes sociales, por lo que, además del impacto tradicional que nos dan las métricas de la Web of Science o Scopus a través de las citas bibliográficas, también podemos ver si un artículo tiene un impacto más allá de ser citado, como puede ser el saber cuántas veces ha sido compartido a través de redes sociales o blogs, especialmente aquellos que son científicos o webs de noticias, como es el caso de infosalut.com.

Podemos encontrar diferentes recursos con los que podemos medir estas métricas alternativas, pero destacan Altmetric y PlumX, así que se incluyó esta información en las referencias bibliográficas publicadas en las noticias de infosalut.com. Cuando se publica la noticia, se debe incluir la información del DOI y/o el PMID para que pueda ser detectada y rastreada por estas herramientas. Esta información también se debe incluir en el tuit, para que éste sea rastreado por las métricas alternativas.

Según el propio Altmetric (2020a), dichas métricas son parte de un grupo de empresas relacionadas con ciencia digital que trabajan conjuntamente para rastrear y analizar la actividad en línea en torno a resultados de investigación académica. Puntúan teniendo en cuenta dónde se haya compartido la información sobre el documento (Altmetric, 2020b). En concreto, será diferente si se ha compartido en Twitter o Facebook o se ha hecho una entrada en Wikipedia o un artículo en un blog. Se representa con un "donut" en el que hay un número y una serie de colores que cambia según el recurso donde se ha difundido.

PlumX (Plum Analytics, 2020) se creó en 2014 y era un producto de EBSCO, pero en 2017 pasó a ser un producto de Elsevier. Tiene en cuenta las citas de Scopus, el uso, si ha guardado, si ha sido mencionado en blogs, en portales de noticias, en redes sociales o en la Wikipedia. A diferencia de Altmetric, se representa con una flor, con unos pétalos que cambian de tamaño según la cantidad de veces que se ha compartido el documento. Hay datos numéricos sobre cuántas veces se ha compartido en Twitter, por ejemplo, pero no hay un valor descargable, por lo que no se ha podido utilizar en la comparativa.

Durante un breve periodo de tiempo Bibliosalut o Infosalut no aportaron impacto a los documentos publicados por los profesionales del SSPIB, porque al compartir la información en redes sociales no se incluía un vínculo 
hacia el artículo en PubMed (en el caso de Bibliosalut se utiliza el vínculo personalizado del resolvedor de enlaces Full Text Finder para que los usuarios puedan ver si está disponible el texto completo o solicitarlo al Servicio de Obtención de Documentos) o el DOI. En 2016 se hizo un análisis sobre cómo se estaba influyendo en el impacto de las referencias cuando se vio que se necesitaba incluir esta información para tener efecto en el número que da Altmetric o en los pétalos de PlumX.

Además, se vio que añadiendo la información con el DOI en la referencia que se pone en la noticia creada en infosalut.com, ésta era rastreada por PlumX y Altmetric (Figuras 6 y 7), lo cual provocaba que se registrara como la publicación de una noticia en una web.

Effect of the comprehensive smoke-free law on time trends in smoking behaviour in primary healthcare patients in Spain: a longitudinal observational study.

Citation Data: BMJ open, ISSN: 2044-6055, Vol: 9, Issue: 3, Page: e020120

Publication Year: 2019

\section{This article has 1 News mention across 1 URL.}

Efecte de la llei integral sobre el tabaquisme en els pacients fumadors d'atenció primària त

4 de septiembre de 2019 | Info Salut $\pi$

La Unitat d'Investigació de la Gerència d'Atenció Primària de Mallorca i

I'Institut d'Investigació Sanitària Illes Balears (IdISBa) han participat en un

estudi observacional longitudinal sobre l'efecte de la llei integral sobre el

tabaquisme en les t...

Read full article $\pi$

Figura 6. Información sobre la noticia publicada en infosalut.com en la página de información de PlumX

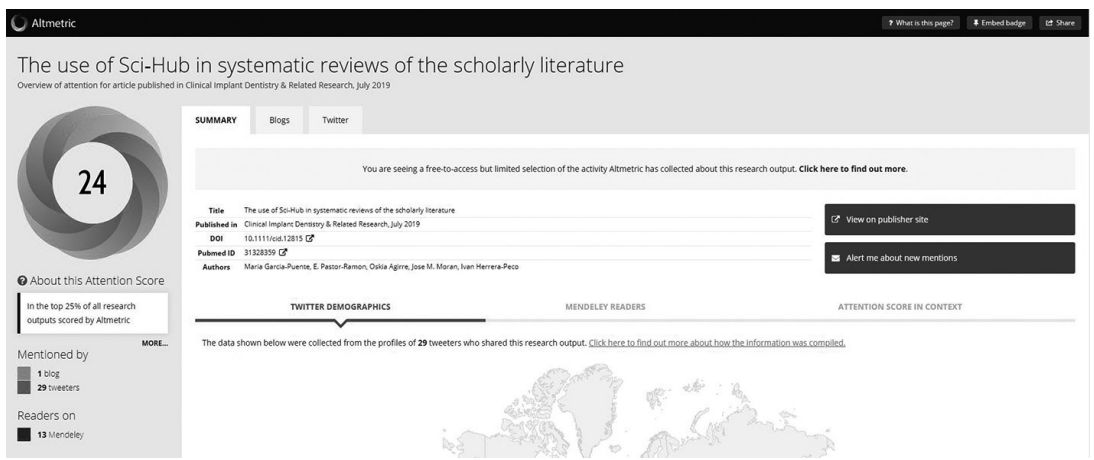

Figura 7. Información sobre la noticia publicada en infosalut.com en la página de información de Altmetric 
Para que el blog o portal de noticias sea rastreado por Altmetric se les debe notificar y lo añaden a un listado del cual no se conoce cuál es su contenido. En cambio, PlumX lo hace automáticamente, desde el momento en el que se añade la información del DOI o el PMID.

\section{Conclusiones}

Esta estrategia que hemos presentado no solamente acerca la biblioteca a los usuarios, ya que, a pesar de que se trata de un portal de noticias de salud, siempre se deja claro que su gestión la realiza el personal de Bibliosalut; asimismo, mejora el impacto social de la producción científica, como demuestra el análisis de datos realizado.

Gracias a este proyecto, hemos dado visibilidad a todo tipo de documentos, y aquellos artículos que se publican en revistas que no ofrecen el DOI, si se hace la noticia en infosalut.com, al menos tienen más posibilidades de ser leídos por otros profesionales. Si no se hiciese esta difusión, al tener algunas revistas presencia en pocas bases de datos y ningún factor de impacto (sobre todo las revistas de enfermería presentes sólo en bases de datos como CuidenPlus), la oportunidad de ser difundidos a través de esta web y después en las redes sociales de Infosalut y Bibliosalut hace que se le dé una visibilidad que no tendrían de otra forma.

El trabajo que se presenta se basa en la difusión que se está realizando desde la biblioteca, el hashtag \#PublicaSalutIB ayuda a poder controlar esos artículos difundidos en un único apartado, de la misma forma que se utilizan otro hashtag para la difusión de formación, por ejemplo.

Debemos tener en cuenta que los objetivos de las bibliotecas van cambiando y en estos momentos en los que baja el número de peticiones de los servicios de obtención de documentos, y que parece que cuesta que se recuperen los presupuestos para suscripciones, la difusión como servicio dado desde la biblioteca puede convertirse en un valor que se añade al que ya tenía la biblioteca como facilitadora del acceso a la información, y que puede ir vinculado a las políticas de Open Access de las instituciones.

Además, se añade el valor de informar a la institución sobre cuál es la producción científica de sus investigadores, ya sea a nivel autonómico, por parte de la Consejería de Salud, como por parte del Servicio de Salud o los centros hospitalarios o atención primaria. Es común que las instituciones no sepan qué se está produciendo en sus centros, a pesar de que se pida en la memoria anual de los diferentes servicios. Además, en muchas ocasiones se desconoce la producción científica porque las instituciones sanitarias no suelen estar disponibles en las webs de los centros. 
Esperamos en un futuro cercano formar a los diferentes servicios en la importancia de hacer difusión de su producción científica a través de las redes sociales, ya que muchos de ellos, aparte de los centros sanitarios, tienen cuentas, especialmente en Twitter, y desde la Consejería de Salud y Consumo del Gobierno de las Illes Balears se está potenciando su uso.

\section{REFERENCIAS}

Allen, Heidi G., Tasha R. Stanton, Flavia di Pietro y G. Lorimer Moseley. 2013. "Social Media release increases dissemination of original articles in the clinical pain sciences". PLoS ONE 8 (7): e68914. https://doi.org/10.1371/journal.pone.0068914

Altmetric. 2020a. "About Us - Altmetric". https://www.altmetric.com/about-us/

Altmetric. 2020b. How is the Altmetric attention score calculated? https://help.altmetric.com/support/solutions/articles/6000060969-how-is-the-altmetric-attention-score-calculated

Buckarma, EeeLN H., Cornelius A. Thiels, Becca L. Gas, Daniel Cabrera, Juliane Bingener-Casey y David R. Farley. 2017. "Influence of Social Media on the dissemination of a traditional surgical research article". Journal of Surgical Education 74 (1): $79-83$. https://doi.org/10.1016/j.jsurg.2016.06.019

Carpenter, Todd A., Nettie Lagace y Sara Bahnmaier. 2016. "Developing standards for emerging forms of assessment: the NISO Altmetrics initiative”. The Serials Librarian 70 (1-4): 85-88. https://doi.org/10.1080/0361526X.2016.1157737

Choo, Esther K., Megan L. Ranney, Teresa M. Chan, N. Seth Trueger, Amy E. Walsh, Ken Tegtmeyer, Shannon O. McNamara, Ricky Y. Choi y Christopher L. Carroll. 2015. "Twitter as a tool for communication and knowledge exchange in academic medicine: a guide for skeptics and novices". Medical Teacher 37 (5): 411-416. https://doi.org/10.3109/0142159X.2014.993371

Costa Marín, Maria, Virgili Páez Cervi, Silvia Sastre Suárez, Elena Pastor Ramon y Mónica Peláez Jiménez. 2011. "Repensando Bibliosalut.Com: nuevo portal de la Biblioteca Virtual de Ciencias de la Salud de Las Illes Balears", en XIV Jornadas Nacionales de Información y Documentación en Ciencias de la Salud, Cádiz, España, 13-15 de abril.

http://hdl.handle.net/10760/15948

Facebook. 2020. "Company Info. About Facebook." https://about.fb.com/company-info/

Fundéu BBVA. 2017. "Etiqueta, mejor que hashtag." https://www.fundeu.es/recomendacion/etiqueta-mejor-que-hashtag-958/

Green, David. 2017. "Academic Search Engine Optimization: ASEO”. Open Journal Systems. 14 de septiembre.

https://openjournalsystems.com/academic-search-engine-optimization/ 
Margaix-Arnal, Dídac. 2008. "Las bibliotecas universitarias y Facebook: cómo y por qué estar presentes." El profesional de la informacion 17 (6): 589-602.

https://doi.org/10.3145/epi.2008.nov.02

Páez, Virgili. 2019. "Bibliosalut, La Biblioteca Virtual de Ciencias de la Salud de las Islas Baleares: 3 lustros en constante evolución”. Trabajo presentado en las XVI Jornadas de Información y Documentación en Ciencias de la Salud, Oviedo, España, 4-5 de abril. http://eprints.rclis.org/34363/

Páez Cervi, Virgili. 2005. "La Biblioteca Virtual de Ciencias de la salud de las Islas Baleares: un proyecto autonómico desde la cooperación bibliotecaria”. E-LIS: EPrints in Library and Information Science. Terrassa. http://eprints.rclis.org/6757/

Páez, Virgili, Elena Pastor-Ramon, Sílvia Sastre-Suárez, Mónica Peláez Jiménez y Maria Costa-Marin. 2013. "Bibliosalut, la Biblioteca Virtual de Ciencias de la Salud de las Islas Baleares”. Boletín de La ANABAD, 1-12. http://hdl.handle.net/10760/23280

Páez, Virgili, Silvia Sastre-Suarez, Catalina Piza, Monica Font y Elena Pastor-Ramon. 2016. "Infosalut: the knowledge management tool of the Public Health System of the Balearic Islands". Trabajo presentado en la 15th EAHIL Conference. Knowledge, Research, Innovation... eHealth!, Sevilla, España, 6-11 de junio. http://eprints.rclis.org/29555/

Pastor-Ramon, Elena, Virgili Páez y Sílvia Sastre-Suárez. 2014. “Gestión de las redes sociales en Bibliosalut”. Trabajo presentado en las XV Jornadas Nacionales de Información y Documentación en Ciencias de la Salud, Madrid, España, 22-23 mayo. http://eprints.rclis.org/23300/

Peset, Fernanda, Antonia Ferrer-Sapena y Tomàs Baiget. 2008. "Evolución social y networking en la comunidad biblio-documental.” El profesional de la informacion 17 (6): 627-636.

https://doi.org/10.3145/epi.2008.nov.05

Plum Analytics. 2020. "About PlumX Metrics". https://plumanalytics.com/learn/about-metrics/

Ramírez Vega, Alexa. 2018. "SEO Académico aplicado a revistas en Acceso Abierto." Trabajo presentado en la Conferencia Internacional BIREDIAL-ISTEC, Lima, Perú, 22-25 octubre.

http://sedici.unlp.edu.ar/handle/10915/71417

Para citar este texto:

Pastor-Ramon, Elena y Virgili Páez. 2021. "Mejora del impacto mediante difusión de la investigación en redes sociales: \#PublicaSalutIB". Investigación Bibliotecológica: archivonomía, bibliotecología e información 35 (88): 29-44.

http://dx.doi.org/10.22201/iibi.24488321xe.2021.88.58355 